\title{
Ventricular Repolarization Intervals in Children Previously Treated with Anthracyclines
}

\author{
Abraham Benatar * Wendy Dewals, Tine Decraene, Arjen Feenstra \\ Department of Pediatric cardiology, Univeristair Ziekenhuis Brussel, Free university of Brussels (VUB), \\ Brussels, Belgium
}

*Corresponding Author: Abraham Benatar, Department of Pediatric Cardiology, Universitair Ziekenhuis (VUB), 101 Laarbeeklaan, 1090 Brussels, Belgium, Email: abraham.benatar@uzbrussel.be

\begin{abstract}
Anthracycline use in childhood cancers may cause dose-dependent cardiotoxicity, which may in the longer term alter cardiac ventricular repolarization. We investigated ventricular repolarization indices in 24 children after completing anthracycline for neoplastic disease as well as 100 healthy age matched individuals as controls. From a 12 lead digital ECG on screen measurements were made. Analytical statistics significance set at $p=<0$, 05. Mean patient age $13 \pm 3.9$ years, controls $13 \pm 4$ years. Time from last anthracycline therapy $5.8 \pm 4.6$ years. Cumulative anthracycline dose: $185-480 \mathrm{mg} / \mathrm{m} 2$. Repolarization intervals patients versus controls respectively: QTc $426 \pm 20 \mathrm{vs} 408 \pm 24 \mathrm{~ms} ;$ JTc $327 \pm 25 \mathrm{vs} 315 \pm 24 \mathrm{~ms}$; TPE dispersion $36 \pm 12$ vs $16 \pm 6 \mathrm{~ms} ;$ QT dispersion $32 \pm 8$ vs $22 \pm 4 \mathrm{~ms}$, maximum TPE $109 \pm 19 \mathrm{vs} 92 \pm$ $14 \mathrm{~ms} ; \mathrm{TPE} \max / Q \bar{T} 0.29 \pm 0.04$ vs $0.25 \pm 0.03 ; \mathrm{TPE} \max / J T \quad 0 . \overline{38} \pm 0.06$ vs $0.33 \pm 0.05$. Repolarization intervals were significantly longer in the anthracycline treated children. Four patients (2 hepatoblastoma, 2 osteosarcoma) developed a dilated cardiomyopathy, one (osteosarcoma) a ventricular tachycardia. Prolongation of repolarization intervals at midterm follow up post -anthracycline, may underlie risk for ventricular arrhythmias or manifestation of clinical/subclinical cardiomyopathy.
\end{abstract}

Keywords: Anthracycline, arrhythmias, QT, QTC, QT dispersion, Tpeak to end, ventricular repolarization, childhood cancers, TPE/QT, TPE/JT, cardiomyopathy

Abbreviations: TPE - T peak to end interval, TPEd - TPE dispersion, QTc - corrected QT interval, SDR spatial dispersion of repolarization, QTd - QT dispersion, JTc - corrected JT interval, ms = milliseconds

\section{INTRODUCTION}

Anthracyclines are used in a wide range of childhood hematologic and solid cancers and known to be associated with dose-dependent cardio toxicity. Anthracyclines work by inhibiting DNA and RNA synthesis, blocking topoisomerase II to prevent DNA and RNA transcription and replication, and creating ironmediated free radicals that damage DNA. Free radical damage to cardiac myocytes is furthermore thought to be the primary mechanism for anthracycline-induced cardiomyopathy [1]. The potential effects of anthracyclines on cardiac repolarization intervals on the electrocardiogram in the medium and longer term after completion of anthracycline in childhood cancer survivors has been previously studied $[2,3]$.
Spatial dispersion of repolarization (SDR) in the form of transmural, transeptal, and apicobasal dispersion of repolarization creates voltage gradients that inscribe the $\mathrm{J}$ wave and $\mathrm{T}$ wave of the ECG. Amplification of this SDR underlies the development of life-threatening ventricular arrhythmias associated with inherited or acquired ion channelopathies giving rise to the long QT, short QT, and Brugada syndromes [46]. The value of the interval between the peak and end of the $\mathrm{T}$ wave as an index of SDR and transmural dispersion of repolarization has been well established [4].

Long-term cancer survivors treated with anthracycline-containing chemotherapy are at risk for serious cardiotoxicity [7] which can be devastating resulting in morbidity, poor quality of life, and premature mortality. Early detection of high-risk patients is considered to be the main 
way to reduce the anthracycline cardiotoxicity. Detection of subclinical cardiac abnormalities or indices may enable fine tuning the antitumor regimens.

We investigated ventricular repolarization indices in children and adolescents after completion of treatment with anthracyclines as part of the treatment regimen for neoplastic disease. We concentrated specifically on Bazett corrected QT interval (QTc), QT dispersion (QTd), T-wave peak to end (TPE) interval, its maximum, its dispersion, TPE/QT, and TPE/JT. These intervals are markers of ventricular repolarization heterogeneity and useful in assessing the potential risk for ventricular arrhythmias [8].

\section{MATERIALS AND METHOdS}

Twenty four patients fully treated with anthracycline(doxorubicin) as part of their treatment protocol for childhood neoplasms and fully recovered were studied at routine outpatient cardlological follow up. Six patients had undergone treatment for acute lymphoblastic leukemia, one for Burkett's lymphoma, two for Hodgkin's lymphoma, three for Ewing's sarcoma, four for osteosarcoma, three for neuroblastoma, three for hepatoblastoma, one for nephroblastoma and one nasopharyngeal carcinoma. The study took place from the beginning of January 2016 to the end of June 2016. At the time of study four patients had previously undergone bone marrow transplantation, three treated for osteosarcoma and one for acute lymphoblastic leukemia. Moreover, at time of study inclusion, four patients were receiving ace-inhibitor therapy (Lisinopril), two in combination with diuretics. Two had previously been treated for hepatoblastoma, and two for osteosarcoma. No patients were hospitalized at time of study. Plasma electrolyte levels, acid-base status and renal function were normal in all four.Concurrently100 healthy age matched subjects were enrolled with informed consent and served as controls.

Criteria for exclusion of control subjects from the study included a family history of arrhythmia or sudden death, a history of acute life-threatening event, bundle branch block on ECG, use of medications known to prolong the
QTinterval or alter the $\mathrm{T}$ wave on ECG, metabolic or central nervous system disorder.

In all those studied, while in a quiet resting state, lying supine, a digital 12leadelectrocardiogram was recorded using a MAC 5500 electrocardiographic system (Marquette Medical Systems, Milwaukee, WI, USA) at a paper speed of $50 \mathrm{~mm} / \mathrm{s}$, sampling rate $1000 \mathrm{~Hz}$. The digital electrocardiograms were stored on a server and subsequently retrieved for analysis. All control subjects were in sinus rhythm and none had a conduction disturbance. The described electrocardiographic parameters were measured using the incorporated digital on screen calipers and were magnified. Parameters included RR interval (average of ten cycles), QT interval from the beginning of the $\mathrm{Q}$ wave to the end of the $\mathrm{T}$ wave, QT dispersion, the difference between the shortest and longest QT interval from the 12 leads, the JT interval, from the beginning of the $\mathrm{J}$ wave to the end of the $\mathrm{T}$ wave, T-peak to $\mathrm{T}$ end intervals (TPE)from the peak of the $\mathrm{T}$ wave to the end of the $\mathrm{T}$ wave (point of return to the isoelectric line, an average from six cycles)and TPE dispersion, the difference between the shortest and longest in the 12 leads. If the Twave amplitude was $<1.5 \mathrm{~mm}$ in a particular lead, or morphologically distorted that lead was excluded from analysis. Additionally, TPE maximum/QT and TPE maximum/JT was calculated for heart rate correction. All measurements were performed bya single investigator $(\mathrm{AB})$. Intra-observer variability and reproducibility was measured for the first ten patients and controls. The coinvestigators randomly assigned electrocardiograms for repeat measurements by the investigator who was blinded to identity.

All patients, excluding the control subjects, underwent two-dimensional and Doppler (pulsed, continuous wave) and tissue Doppler (TDI) echo cardiographic examination for assessment of left ventricular (LV) diastolic and systolic function. For the left ventricular systolic function the assessment of left ventricular ejection and shortening fraction utilizing the Simpson Rule for determining ventricular volumes and Teicholz methods were utilized [9:10]. An apical four- and twochamber view was recorded from which the endocardial border was outlined in end-diastole 
and end-systole. An M-mode linear measurements of the ventricles was also performed and recorded. Pulsed Doppler mitral peak $\mathrm{E}$ and $\mathrm{A}$ wave velocity $(\mathrm{cm} / \mathrm{sec})$, mitral valve E/A ratio, pulsed wave TDI e', mitral E/e', a' were recorded and measured. . For the older patients the four variables for identifying diastolic dysfunction and their abnormal cutoff values utilized were annular e' velocity: septal e' $<7 \mathrm{~cm} / \mathrm{sec}$, lateral e' $<10 \mathrm{~cm} / \mathrm{sec}$, average $\mathrm{E} / \mathrm{e}^{\prime}$ ratio $>14$, LA volume index $>34 \mathrm{~mL} / \mathrm{m}^{2}$, and peak TR velocity> $2.8 \mathrm{~m} / \mathrm{sec}$ for the older patients 9;10].Additionally, mitral annular plane systolic excursion (MAPSE) was measured which we utilized as a surrogate of the left ventricular longitudinal function. Measurements were done with $\mathrm{M}^{-}$mode beam positioned on the lateral mitral annulus, in line with the left ventricular long axis.

\subsection{Statistics}

Statistical analyses were carried out with a package of SPSS IBM software version 24 (USA). Kolmogorov-Smirnov test was used to check the Gaussian distribution of continuous variables. Continuous variables were presented as mean \pm standard deviation, median and range. Categorical variables were presented as number and frequencies .The Student's t-test and analysis of variance were used to compare continuous variables as appropriate. Additionally, Fisher's exact test, was used to compare categorical variables. Significance was considered at a $P$ value of $\leq 0.05$. An odds ratio was calculated for risk of development of cardiomyopathy.
The study was conducted in a single tertiary referral center.

The study was approved by the medical ethics committee of the Academic Hospital of the Free University of Brussels (VUB).

\section{RESULTS}

The demographic data are shown in Table 1. The mean age of the patients and controls are comparable. Males marginally outnumbered females in both the control and patient groups. The mean time from the last anthracycline therapy was $5.8+4.6$ (median 5.9) years. The cumulative anthracycline dose ranged from 185 - $480 \mathrm{mg} / \mathrm{m} 2$.Of the four patients who underwent bone marrow transplantation, only one had received total body irradiation at a maximum dose of 21 Gray. The three Ewing's sarcoma patients received in addition, irradiation therapy to the affected tumor region, sparing the heart region. Measured repolarization intervals are shown in Table 2. The patient group intervals were significantly longer than those of controls, $\mathrm{p}<0,05$. QTcBazett and JTc were significantly longer in the patients when compared to controls. JTc was calculated to eliminate the variation in QRS duration. Similarly TPE and QT dispersion were significantly prolonged in the patient group as was maximum TPE (Table 2, Figure 1). Even when TPE maximum was corrected by QT and JT intervals the ratio prolongation persisted in the patient group.

Table1. Demographic data of studied patients and normal controls

\begin{tabular}{|l|l|l|}
\hline & Controls & Patients \\
\hline Number & 100 & 22 \\
\hline Age(years) & $13 \pm 4.3(13.8)$ & $13 \pm 3.9(13)$ \\
\hline Gender $\mathrm{m} / \mathrm{f}$ & $56 / 44$ & $14 / 8$ \\
\hline
\end{tabular}

$m=$ male, $f=$ female, parentheses $=$ median, $\pm=$ standard deviation

Table2. Measured Intervals of Patients and Normal Controls

\begin{tabular}{|l|l|l|}
\hline Intervals (ms) & Controls & Patients \\
\hline QTc & $408 \pm 24(370-470)$ & $426 \pm 21(396-476)^{*}$ \\
\hline JTc & $315 \pm 24(276-356)$ & $327 \pm 24(285-364)^{*}$ \\
\hline TPE dispersion & $16 \pm 6(10-30)$ & $36 \pm 12(14-66)^{*}$ \\
\hline QT dispersion & $22 \pm 4(12-30)$ & $32 \pm 8(12-38)^{*}$ \\
\hline Maximum TPE & $92 \pm 14(60-118)$ & $109 \pm 17(76-146)^{*}$ \\
\hline TPE max/QT & $0.25 \pm 0.03(0.17-0.3)$ & $0.29 \pm 0.04(0.22-0.4)^{*}$ \\
\hline TPE max/JT & $0.33 \pm 0.05(0.21-0.39)$ & $0.38 \pm 0.06(0.28-0.52)^{*}$ \\
\hline
\end{tabular}

$m s=$ millisecondes $, *=p<0,05, \pm=$ standard déviation, parantheses $=$ range 


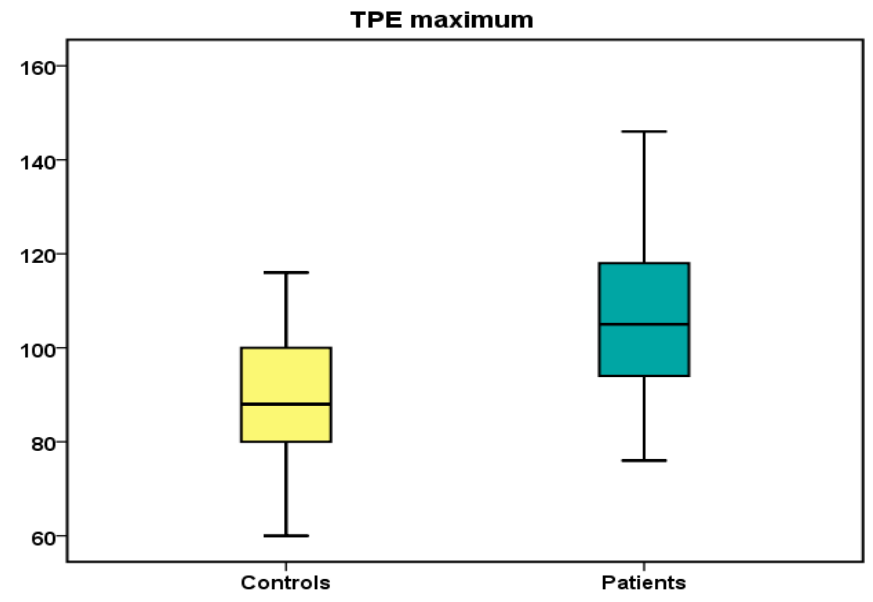

Figure1. Box plot of TPE maximum in milliseconds comparing patients and controls. Horizontal bars on top and bottom of whiskers represent the limits. Horizontal bar in the box represents the median; horizontal bar top of box represents upper quartile value, bottom of the box lower quartile value. Y axis = values in milliseconds.

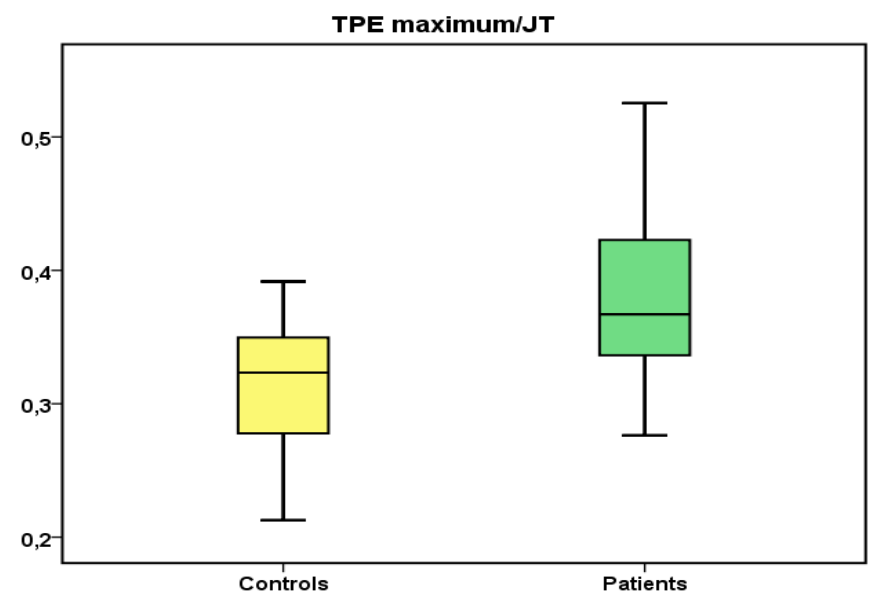

Figure2. Box plot of TPE maximum JT ratio. Horizontal bars on top and bottom of whiskers represent the limits. Horizontal bar in the box represents the median, horizontal bar top of box represents upper quartile value, bottom of the box lower quartile value.

Echo cardiographic examination was evaluated as normal in 20 patients. Four patients developed a dilated cardiomyopathy, one with very severe global left ventricular dysfunction of all segments (Left ventricular end diastolic dimension $\geq 6.9 \mathrm{~cm} / \mathrm{m} 2$, ejection fraction $<30$ $\%$, and left atrial volume $>48 \mathrm{~mL} / \mathrm{m} 2$,TDI annular e' velocity: septal e' $3 \mathrm{~cm} / \mathrm{sec}$, lateral e' $5 \mathrm{~cm} / \mathrm{sec}$, average E/e' ratio 18 . Following the study this patient deteriorated further and underwent heart transplantation. The longest repolarization intervals were observed in this patient. In 2 other patients the LV was moderately dilated (Left ventricular end diastolic dimension $3.5-3.6 \mathrm{~cm} / \mathrm{m} 2$ ) ejection fraction 45 and 50 per cent respectively. Another patient had a LV end diastolic dimension $3.2 \mathrm{~cm} / \mathrm{m} 2$ with hypokinesia of the anteroseptal segment.
A previously treated osteosarcoma fifteen year old patient who was 2.5 years post completion of anthracycline treatment, complained of excessive sweating and dyspnea on minimal exertion. A 24 hourHolter examination revealed runs of ventricular tachycardia with left bundle branch block morphology in addition to numerous premature ventricular contractions (PVCs) of the same morphology. An electrophysiological study identified an arrhythmogenic focus which was mapped to the posterior right ventricular outflow. A successful radio-frequency ablation of the focus was performed. No recurrences of ventricular tachycardia have since occurred.

A hepatoblastoma patient treated in infancy, presented with severe dilated cardiomyopathy at the age 10 years, which progressed despite maximal treatment with diuretics, angiotensin I converting enzyme inhibitor and carvedilol. Left 
ventricular function was globally depressed (same patient described previously under echocardiographic examination) with dyskinesia. SF was estimated at 15 per cent and left ventricular ejection fraction <30 percent. Total anthracycline dose administered was 377 $\mathrm{mg} / \mathrm{m} 2$.After inclusion in the study he developed left bundle branch block with progressively increasing QRS prolongation, worsening left ventricular dyskinesia and poor cardiac function. $\mathrm{He}$ underwent resynchronization therapy with no objective beneficial clinical or echocardiographic effect. Heart transplantation was subsequently performed.

The calculated odds ratio for the development of a dilated cardiomyopathy was 0.2 with $95 \%$ confidence interval of 0.0660 to 0.606 , significance level $\mathrm{p}=0.0044$.

Intra observer measurement variability: The maximum difference in measurements did not exceed8 milliseconds for TPE, 15 milliseconds and 12 milliseconds for QT and JT intervals respectively and 15 milliseconds for $\mathrm{RR}$ intervals.

\section{CONCLuSion}

The purpose of this study was to assess ventricular repolarization in anthracycline treated children a number of years after completing their treatment by way of surrogate ectrocardiographic indices, namely QT dispersion, QTc, JTc, TPE dispersion and TPE ratios to correct for effect of heart rate. The anthracycline treated group had the most prolonged measured indices of ventricular repolarization. This may represent a surrogate marker of underlying subclinical cardiomyopathy. Notably only a single patient from a cohort of 24 developed an overt significant rhythm disorder namely a ventricular tachycardia. Lack of continuous monitoring limited our detection capacity which may be an important shortcoming.

Notably the patient with the worst cardiac contractile function and the longest ventricular repolarization intervals, went on to develop a left bundle branch block though ventricular arrhythmias were not detected. He was on vasodilator, diuretic and beta blocker medical therapy which may have provided arrhythmia protection.

Back in 1992 researchers [11] discovered that the use of anthracycline in patients could cause QTc prolongation, increased supraventricular, and ventricular tachycardia compared to normal controls $(\mathrm{P}<0.001)$. Contrary to our study these researchers found no significant difference between the case and the control group regarding QT dispersion and QTc dispersion $(\mathrm{p}>0.05)$. A possible drawback in that study was the lack of more accurate digital measurements. More recent studies have reinforced the association between anthracycline and prolonged corrected QT (QTc) intervals as measured on surface the surface electrocardiogram, which may point to heightened risk of ventricular tachycardia $[12,13]$ Anthracyclines have been directly associated with premature ventricular contractions, sinus node dysfunction, ventricular late potentials, and decreased QRS voltage. The incidence of such occurrence ranges from $10 \%$ to $30 \%[14,15]$. The mechanism and the structural characteristics of drugs most likely to alter the duration of the QT interval have been determined. The QT interval is presumed to increase when HERG (human ether-a-go-go) potassium channel activity (delayed rectifier potassium current) is altered. By interfering with these channels, drugs slow potassium ion entry into the myocyte and thereby prolong repolarization [16]. Additional mechanisms may also be involved, since not all drugs that impede HERG potassium channel activity induce QT prolongation. Functionally, neither QT prolongation nor HERG blockadeis an absolutely reliable indicator of arrhythmogenic risk. As with QT prolongation, HERG inhibition is ultimately merely a "marker" for risk. It is widely accepted that HERG liability is neither absolutely necessary nor sufficient for a drug to have a risk for the genesis of torsade des pointes [17]. Both adults and children with cancer may be more susceptible to QT prolongation because of comorbid conditions, disease-related electrolyte disturbances, or concomitant treatment with potentially proarrhythmic medications [18].

QT interval and its correction (QTc) have been used to predict cardiac arrhythmias, while QT dispersion as a marker has also been implemented in clinical practice. The QT dispersion represents the difference between the maximum and minimum QT interval on the twelve lead surfaces ECG and depicts the heterogeneity state of ventricular repolarization. An increase in ventricular heterogeneity augments the vulnerable period time in the heart and predisposes to ventricular arrhythmias [19]. In this study the anthracycline treated patients 
exhibited significantly greater QT dispersion as compared to controls.

JTc intervals were calculated in order to eliminate the variation of QRS duration on the interpretation of ventricular repolarization. JTc intervals interpretation follows similar philosophiesto QTc interpretation. In this study, similar to the QTc values, the anthracycline group of patients had significantly prolonged JTc values.

TPE is the resultant of the global distribution of the repolarization process and is a surrogate diagnostic parameter. $\mathrm{T}$ wave morphology descriptors are sensitive to drug-induced changes and maybe a useful addition to the QT interval in cardiac safety trials. The precordial leads are unipolar leads placed on the chest, which are referenced to Wilson central terminal. The direction of these leads is radially outward from the "center" of the heart, the center of the Einthoven triangle. Unlike the precordial leads, the bipolar limb leads, including leads I, II, and III, do not look across the ventricular wall. While TPE intervals measured in these limb leads may provide an index of transmural dispersion in repolarization, they are more likely to reflect global dispersion, including a picobasal and interventricular dispersion of repolarization [20 -22]. Anearlier study showed that global TPE can be a strong indicator of transmural dispersion in depolarization [21].

Delayed effects of doxorubicin administration were studied in the rat to determine repolarization heterogeneity of the ventricular epicardium [23]. Doxorubicin-induced inhomogeneous prolongation of repolarization of the ventricular epicardium which in turn resulted in increasing heterogeneity of ventricular repolarization because of increasing intraregional heterogeneity while interregional differences were lost. In the rat right ventricular repolarization was more sensitive to doxorubicin than left ventricular repolarization [23].This is of particular interest as one of our study patients developed right ventricular outflow (RVOT) tachycardia. The association may be more than pure conjecture. In a multicenter prospective study evaluating 90 patients with frequent premature ventricular contractions originating from the RVOT, 24 with metabolic syndrome (MS) and 66 without, who underwent radio frequency ablation, patients with MS showed a higher acute post-procedural recurrence of right outflow tract PVCs [24]. In the metabolic syndrome there is some direct or indirect influence on ventricular electrophysiology by different action pathways which alterthe ionic channels conduction properties. The higher prevalence of obesity, dyslipidemia, hypertension and diabetes leads MS patients to have an augmented oxidative stress and arhythmogenesis, with abnormalities in sympatho-vagal balance, QT interval and QT dispersion and a fibrotic and adipotic cardiac conduction system involvement [24]. In the metabolic syndrome a "metabolic pro arrhythmic effect" may alter the calcium channel activity, leading to QT interval prolongation [25]. The QT interval prolongation may in turn trigger ventricular arrhythmias. The arrhythmic burden in MS may be directly related to alterations in oxido-reductive, inflammatory tone, and sympathetic activity [25] which may lead to a pro-arrhythmic state, which may have prognostic consequences. The patient cohort we studied did not have any features nor evidence of the metabolic syndrome. Nonetheless, some underlying pathogenesis mechanistic similarities could theoretically be drawn between the metabolic syndrome and anthracycline cardiomyopathy.

We are unable to disregard the compounding role concomitant drugusein four patients (Lisinopril and diuretics) may have had on myocardial channel function and thus influence repolarization. Serum electrolytes and acid-base status was normal in all patients at time of study.

TPE interval and TPE/QT ratio have been used as new ECG markers of increased dispersion of ventricular repolarization. Prolongation of the TPE interval has been identified to be related with ventricular arrhythmia and sudden cardiac death [26-30]. Electrophysiological studies have shown that TPE interval prolongation is associated with ventricular tachycardia induction and the spontaneous occurrence of ventricular tachycardia [21, 22]. Furthermore, the TPE/QT ratio is considered to be a more sensitive index of arhythmogenesis when compared with the solitary use of either the TPE or QT intervals, as it is neither affected by variations in body weight nor heart rate [26].

A few studies have considered the incidence and importance of cardiac arrhythmias long after chemotherapy during childhood. Some have reported occasional instances of sudden death that may be related to repolarization abnormalities (QT prolongation and abnormal QT dispersion) in association with ventricular 
dysfunction [31], others have described only infrequent and clinically unimportant arrhythmias [32]. Other studies of pediatric cancer survivors have described ventricular arrhythmias in association with late ventricular dysfunction [7], though in general, these studies have been small and did not systematically evaluate the population at risk. There are no data addressing the issue of whether the risk of arrhythmia exceeds that found in other causes of ventricular dysfunction.

Anthracyclines are the best-known class of chemotherapeutic drugs associated with cardiotoxicity [18]. These agents (especially doxorubicin) have long been an important component of therapy for hematological and solid tumors in children.

There is no 'safe' anthracycline dose if the goal is normal long-term cardiovascular status but higher lifetime cumulative doses of anthracycline, higher dose rates, female sex, longer follow-up, younger age at anthracycline treatment, pre-existing cardiovascular disease, and cardiac irradiation are associated with more severe cardiotoxicity[18].

The pathophysiology of ventricular arrhythmias in non-ischemic dilated cardiomyopathy (NIDCM) is less well understood. Arrhythmogenesis is likely multifactorial and may be related to structural changes such as fibrosis and left ventricular dilation as well as to primary and secondary electrophysiological changes; these may result in ventricular tachyarrhythmia's due to reentry, abnormal automaticity, and triggered activity. Focal mechanisms seem to underlie the isolated premature ventricular complexes (PVCs) and non-sustained ventricular tachycardia (VT) that originate in the sub endocardium [33]. Mechanism of occurrence of sustained monomorphic VT in NIDCM, is most commonly due to reentry within the myocardium [34, 35]. Similar to ischemic cardiomyopathy, the substrate for reentry in NIDCM is probably scar-based [36]. Recent magnetic resonance imaging data confirm that the presence low-voltage electrograms along the reentry circuit is consistent with scar [36]. The pathogenesis of polymorphic VT and ventricular fibrillation in NIDCM is less understood. A meta-analysis of forty five studies to estimate the performance of 12 commonly reported risk stratification tests as predictors of arrhythmic events in patients with non ischemic dilated cardiomyopathy concluded that a combination of tests would be required to optimize risk stratification in this population and may be due to the interplay of several variables and that no single abnormality can fully explain the process [34].

Late-onset cardiomyopathy is a serious consequence of anthracycline therapy resulting in death or the need for cardiac transplantation in some patients. Unlike most other forms of dilated cardiomyopathy, the major pathological changes appear to be interstitial and/or replacement fibrosis which can be diffuse or multifocal without significant cardiac hypertrophy [37].

Telemonitoring high risk anthracycline treated children for arrhythmias may prove to be beneficial. Telemonitoring is a safe and efficient monitoring system for internal cardioverter defibrillator device (ICD) recipients. It has proved its importance in predicting heart failure hospitalization in patients treated with chronic resynchronization therapy with defibrillator in the adult population [38]. In our cohort of patients studied there were no ICD recipients.

The early detection of anthracycline induced cardiomyopathy (AIC) may be made by biomarker detection, echocardiographic followup of left ventricular systolic and diastolic function, and cardiac enzymes [39].According to several clinical studies in asymptomatic survivors of acute leukemia, the N-terminal of the prohormone brain natriuretic peptide (NTproBNP) is a sensitive cardiac biomarker, which proved to be more useful than cardiac troponin I in the early identification of high-risk patients with AIC [40]. Moreover, in childhood cancer survivors, growth-differentiation factor-15 level may be used as a biomarker of AIC [41]. There is clinical evidence verifying that echocardiography, radionuclide ventriculo graphy (multigated acquisition (MUGA) scan) and cardiac magnetic resonance imaging (MRI) are important strategies for analyzing subclinical late AIC in pediatric patients [42].

Echocardiography is a non-invasive investigative method commonly used for monitoring AIC. Reduced LV systolic strain after anthracycline treatment may indicate early impairment of myocardial function, before any detectable change in left ventricular ejection fraction (LVEF) [43].Peak systolic strain rate appears to be more useful than TDI velocities to evaluate left ventricular dynamics during volume loading in patients with depressed left ventricular function. As it reduces significantly with volume loading in the absence of change in 
LVEF or peak systolic velocities at TDI. Strain rate is a relative load-independent index of systolic function, the reduction observed is probably related to the decrease in left ventricular systolic performance that follows volume loading in heart failure patients [44]. In this study we did not perform strain imaging.

Furthermore, in another clinical trial, dobutamine-stress QT dispersion and heart-rate corrected QT dispersion were also helpful for detecting AIC and subclinical cardiac abnormality at low cumulative anthracycline doses [67].

The present study provides some insights in ventricular repolarization intervals in the medium term follow up of patients after anthracycline for childhood cancers. The prolonged repolarization intervals observed represent dispersion in ventricular repolarization. In four patients cardiac systolic dysfunction was evident, though the majority were free of left ventricular dysfunction. The major portion of the anthracycline treated patients displayed prolonged ventricular repolarization intervals in the absence of clinical and echocardiographic evidence of systolic and diastolic dysfunction.

We hypothesize that pronged ventricular repolarization may reflect a form of subclinical cardiomyopathy and could be a surrogate marker. Additional prospective studies with a larger cohort of patients are required to further elucidate this finding.

Future prospective and multicenter randomized clinical trials are needed to identify new strategies of managing the risk factors and to evaluate new methods for early anthracycline induced cardiomyopathy detection. Furthermore, a major target for pharmaceutical research ought to be the discovery of new anthracycline characterized by less cardiotoxicity for improving the life quality and expectancy of cancer survivors.

\section{STUDY LIMITATIONS}

Several limitations need to be acknowledged and emphasized. Foremost, the study comprised a small sample size of patients, under 100, which may compromise the statistics. The statistics need to be interpreted accordingly. Patient selection bias from a single tertiary referral center may be an unintended limitation. A range of medical therapy was used in a few patients and the interaction of medical therapy with the prognostic value of these tests may be a significant factor. The study period provided a smalltime window to assess cardiac rhythm. With the current advanced technology new systems of Telemonitoring could provide valuable information regarding incidence of PVCs or more serious arrhythmias in this cohort of patients. The follow-up duration may have been too short to fully characterize the incidence of lethal arrhythmias in the more long term in anthracycline treated children. .

\section{REFERENCES}

[1] Sawyer DB. Anthracyclines and heart failure. N Engl J Med 2013; 368:1154-1156.

[2] Amoozgar H, Zareifar S, Borzoie M, Qasem F.Heart repolarization changes after anthracycline therapy in the children with cancer. Iran J Ped Hematol Oncol. 2014; 4:103108

[3] Uchikoba Y1, Fukazawa R, Ohkubo T, Maeda $\mathrm{M}$, Ogawa S.Early detection of subclinical anthracycline cardiotoxicity on the basis of QT dispersion. J Nippon Med Sch. 2010:234-43.

[4] Antzelevitch C. Drug-induced spatial dispersion of repolarization.Cardiol J. 2008; 15:100-121.

[5] Xue J, GaoW, Chen Y, et al. Study of repolarization heterogeneity and electrocardiographic morphology with a modelingapproach. J Electrocardiol. 2008; 41:581-587.

[6] Yan GX, Antzelevitch C. Cellular basis for the normal Twave and the electrocardiographic manifestations of the long-QT syndrome. Circulation. 1998; 98:1928-1936.

[7] Bansal N, Amdani S, Lipshultz ER, Lipshultz SE. Chemotherapy-induced cardiotoxicity in children. Expert Open Drug Metab Toxicol. 2017; 8:817-832.

[8] Porthan K, Viitasalo M, Toivonen L. Predictive value of electrocardiographic Twave morphology parameters and T-wave peak to $\mathrm{T}$-wave end interval for sudden cardiac death in the general population. Circ Arrhythm Electrophysiol 2013; 6(4):690-696.

[9] NaguehSF et al. Recommendations for the Evaluation of LeftVentricular Diastolic Function by Echocardiography:An Update from the American Society of Echocardiography and the European Association of Cardiovascular Imaging. J Am Soc Echocardiogr. 2016; 29:277-314.

[10] Lang RM, Badano LP, Mor-Avi V, Afilalo J, Armstrong A, Ernande L, et al. Recommendations for cardiac chamber quantification by echocardiography in adults: An update from the American Society of Echocardiography and the European 
Association of Cardiovascular Imaging. J Am Soc Echocardiogr. 2015; 28:1-39-e14.

[11] Larsen RL, Jakacki RI, Vetter VL, Meadows AT,Silber JH, Barber G. Electrocardiographic changesand arrhythmias after cancer therapy in children andyoung adults. Am J Cardiol. 1992; 1; 70(1):73-77.

[12] Bagnes C, Panchuk PN, Recondo G. Antineoplastic chemotherapy induced QTc prolongation. Curr Drug Saf. 2010; 5:93-96.

[13] Albini A, Pennesi G, Donatelli F, Cammarota R, De Flora S, Noonan DM.Cardiotoxicity of anticancer drugs: the need for cardio-oncology and cardio-oncological prevention. J Natl Cancer Inst. 2010; 102:14-25.

[14] Pratila MG, Steinherz LJ, Pratilas V. Sick sinus syndrome in a teenagertreated with idarubicin. J Cardiothorac Vasc Anesth. 1993; 7:125-126.

[15] Kilickap S, Akgul E, Aksoy S, Aytemir K, Barista I. Doxorubicin-inducedsecond degree and complete atrioventricular block. Europace. 2005; 7:227-230.

[16] Strevel EL, Ing DJ, Siu LL. Molecularly targeted oncology therapeuticsand prolongation of the QT interval. J Clin Oncol. 2007; 25:3362-3371.

[17] Witchel HJ.Drug-induced hERG Block and Long QT Syndrome. Cardiovascular Therapeutics. 2011; 29: 251-259.

[18] Lipshultz SE et al. Long-term Cardiovascular Toxicity in Children,Adolescents, and Young Adults Who Receive CancerTherapy: Pathophysiology, Course, Monitoring,Management, Prevention, and Research Directions. A Scientific Statement from the American Heart Association. Circulation. 2013; 128:1927-1995.

[19] Antzelevitch C, Shimizu W, Yan GX, Sicouri S. Cellular basis for QT dispersion. J Electrocardiol. 1998; 30 Suppl: 168-175.

[20] Antzelevitch C. Drug-induced spatial dispersion of repolarization.Cardiol J. 2008; 15:100-121.

[21] Xue J, GaoW, Chen Y, et al. Study of repolarization heterogeneityand electrocardiographic morphology with a modelingapproach. J Electrocardiol. 2008; 41: 581-587.

[22] Opthof T, Coronel R,Wilms-Schopman FJ, et al. Dispersionof repolarization in canine ventricle and the electrocardiographicT wave: $\mathrm{T}(\mathrm{p}-\mathrm{e})$ interval does not reflect transmuraldispersion. Heart Rhythm. 2007; 4:341-348

[23] Kharin SN, Krandycheva VV, Strelkova MV, Tsvetkova AS, Shmakov DN.Doxorubicininduced changes of ventricular repolarization heterogeneity: results of a chronic rat study. Cardiovasc Toxicol. 2012; 4:: 312-317.
[24] Sardu C, et al. Metabolic syndrome is associated with a poor outcome in patients affected by outflow tract premature ventricular contractions treated by catheter ablation. BMC Cardiovascular Disorder. 2014; 14:176.

[25] Sardu C, et al. Cardiac electrophysiological alterations and clinical response in cardiac resynchronization therapy with a defibrillator treated patients affected by metabolic syndrome. Medicine (Baltimore). 2017; 96(14):e6558

[26] Gupta P, Patel C, Patel H et al. T(p-e)/QT ratio as an index of arrhythmogenesis. J Electrocardiol, 2008; 41: 567-574.

[27] Kors JA, Ritsema van Eck HJ, van Herpen G. The meaning of the Tp-Te interval and its diagnostic value. J Electrocardiol. 2008; 41: 575-580.

[28] Smetana P, Schmidt A, Zabel $M$ et al. Assessment of repolarization heterogeneity for prediction of mortality in cardiovascular disease: Peak to the end of the $\mathrm{T}$ wave interval and nondipolar repolarization components. J Electrocardiol, 2011; 44: 301-308.

[29] Erikssen G, Liestøl K, Gullestad L, Haugaa $\mathrm{KH}$, Bendz B, Am-lie JP. The terminal part of the QT interval ( $\mathrm{T}$ peak to $\mathrm{T}$ end): A predictor of mortality after acute myocardial infarction. Ann Noninvasive Electrocardiol. 2012; 17: 8594.

[30] Panikkath R, Reinier K, Uy-Evanado A et al. Prolonged Tpeak-to-Tend interval on the resting ECG is associated with increased risk of sudden cardiac death. Circ Arrhythm Electrophysiol, 2011; 4: 441-447.

[31] Gupta M, Thaler HT, Friedman D, Steinherz L. Presence of prolonged dispersion of QT intervals in late survivors of childhood anthracycline therapy [published correction appears in Pediatr Hematol Oncol. 2007;24:649]. Pediatr Hematol Oncol. 2002;19:533-543

[32] Postma A, Elzenga NJ, Haaksma J, SchasfoortVan Leeuwen MJ, Kamps WA, Bink-Boelkens MT. Cardiac status in bone tumor survivors up to nearly 19 years after treatment with doxorubicin: a longitudinal study. Med Pediatr Oncol. 2002; 39::86-92.

[33] Pogwizd SM, McKenzie JP, Cain ME. Mechanisms underlying spontaneous and induced ventricular arrhythmias in patients with idiopathic dilated cardiomyopathyCirculation, 1998; 98: 2404-2414.

[34] Goldberger JJ, Subačius H, Patel T, Cunnane R, Kadish AH. Sudden cardiac death risk stratification in patients with nonischemic dilated cardiomyopathy. J Am Coll Cardiol. 2014; 63:1879-1889.

[35] Delacretaz E, Stevenson WG, Ellison KE, Maisel WH, Friedman PL. Mapping and 
radiofrequency catheter ablation of the three types of sustained monomorphic ventricular tachycardia in nonischemic heart disease. $\mathrm{J}$ Cardiovasc Electrophysiol. 200; 11:11-17.

[36] HsiaHH, Callans DJ, Marchlinski FE. Characterization of endocardial electrophysiological substrate in patients with nonischemic cardiomyopathy and monomorphic ventricular tachycardia. Circulation. 2003; 108:704-710.

[37] Bernaba BN, Chan JB, Lai CK, Fishbein MC.Pathology of late-onset anthracycline cardiomyopathy.Cardiovasc Pathol. 2010; 19:308-311.

[38] Sardu C et al. Telemonitoring in heart failure patients treated by cardiac resynchronisation therapy with defibrillator (CRT-D): the TELECART Study.Int J Clin Pract. 2016; 70(7):569-576.

[39] Oztarhan K, Guler S, Aktas B, Arslan M, Salcioglu Z, Aydogan G. The value of echocardiography versus cardiac troponin I levels in the early detection of anthracycline cardiotoxicity in childhood acute leukemia: prospective evaluation of a 7-year-long clinical follow-up. Pediatr Hematol Oncol. 2011; 28:380-394.

[40] Stachowiak P, Kornacewicz-Jach Z, Safranow K. Prognostic role of troponin and natriuretic peptides as biomarkers for deterioration of left ventricular ejection fraction after chemotherapy. Arch Med Sci. 2014; 10:10071018.

[41] Arslan D, Cihan T, Kose D, et al. Growthdifferentiation factor-15 and tissue Doppler imaging in detection of asymptomatic anthracycline cardiomyopathy in childhood cancer survivors. Clin Biochem. 2013; 46:1239-1243.

[42] Basar EZ, Corapcioglu F, Babaoglu K, Anik Y, Gorur Daglioz G, Dedeoglu R. Are cardiac magnetic resonance imaging and radionuclide ventriculography good options against echocardiography for evaluation of anthracycline induced chronic cardiotoxicity in childhood cancer survivors? Pediatr Hematol Oncol. 2014; 31:237-252.

[43] Stoodley PW, Richards DA, Hui R, et al. Twodimensional myocardial strain imaging detects changes in left ventricular systolic function immediately after anthracycline chemotherapy. Eur J Echocardiogr. 2011; 12:945-952.

[44] Vicario ML et al. Effects of volume loading on strain rate and tissue Doppler velocity imaging in patients with idiopathic dilated cardiomyopathy. J Cardiovasc Med (Hagerstown). 2006; 7(12):852-858

[45] Uchikoba Y, Fukazawa R, Ohkubo T, Maeda M, Ogawa S. Early detection of subclinical anthracycline cardiotoxicity on the basis of QT dispersion. J Nippon Med School. 2010; 77:234-243.

Citation: Abraham Benatar, Wendy Dewals, Tine Decraene \& Arjen Feenstra. Ventricular Repolarization Intervals in Children Previously Treated with Anthracyclines. ARC Journal of Cardiology. 2017; 3(2) 6-15. doi: dx.doi.org/11.20431/2455-5991.0302002.

Copyright: (C) 2017 Authors. This is an open-access article distributed under the terms of the Creative Commons Attribution License, which permits unrestricted use, distribution, and reproduction in any medium, provided the original author and source are credited. 